\title{
Modelamiento Termodinámico para la Inhibición de Incrustaciones de Carbonato de Calcio en Sistemas Hidrocarburos.
}

\section{Thermodynamic Models to Inhibit Calcium Carbonate Scale in Hydrocarbon Systems.}

\author{
Carmen Pinzón Torres ${ }^{1}$, Lucas Santiago García Ortiz ${ }^{2}$ y Diego Felipe Rodríguez Osorio ${ }^{3}$
}

\section{Resumen}

El presente trabajo se basa en el modelamiento termodinámico de la inhibición de incrustaciones para generar una herramienta de cómputo que permita estimar el carácter incrustante del agua de formación, la eficiencia y concentración mínima de los inhibidores, al igual que la fijación y el avance de éstos, para las condiciones específicas del sistema donde se implemente el tratamiento de inhibición. Scale Inhibition CaCO3 es un software diseñado y programado exclusivamente para la aplicación en campos petroleros y es amplio en la predicción, control, tratamiento y manejo de las incrustaciones de carbonato de calcio. Permite predecir la concentración mínima y eficiente de inhibidor necesario para las condiciones específicas de un pozo y seleccionar el inhibidor más eficiente para la aplicación de un tratamiento "squeeze". También pronostica la cantidad y costo de inhibidor necesario para inhibir, en un radio de acción predeterminado.

Palabras claves: Incrustaciones de carbonato de calcio; precipitación de minerales; modelamiento termodinámico de inhibición; tratamiento “squeeze”.

\begin{abstract}
This paper is based on thermodynamic modeling of the inhibition of scale to generate a computational tool to estimate the fouling character of formation water, efficiency and minimum concentration of inhibitors, like the setting and advance their , for the specific conditions of the system which implements the inhibition therapy.

Scale Inhibition CaCO3 is software designed and programmed exclusively for use in oil fields and is broad in the prediction, monitoring, treatment and management of calcium carbonate scale. To predict the minimum concentration of inhibitor necessary and efficient for the specific conditions of a well and select the most efficient inhibitor for the treatment application squeezed. It also predicts the amount and cost of inhibitor necessary to inhibit, in a predetermined radius.
\end{abstract}

Keywords: Scale calcium carbonate; mineral precipitation; thermodynamic modeling inhibition; squeeze treatment.

1 Química, Especialista en Sistemas Dinámicos, Docente de tiempo completo. Universidad Surcolombiana Neiva. Avenida Pastrana Carrera $1^{a}$, carpinto@usco.edu.co

2 Ingeniero de Petróleos. Universidad Surcolombiana Neiva. Avenida Pastrana Carrera 1․ Isantiagogarcia@hotmail.com

3 Ingeniero de Petróleos. Universidad Surcolombiana Neiva. Avenida Pastrana Carrera 1ª.diegofelipe.0323@gmail.com 


\section{Introducción}

Las incrustaciones (scales) son depósitos adherentes y duros que se forman por procesos de depositación de compuestos inorgánicos, a partir de soluciones acuosas sobresaturadas de minerales conocidas como salmueras, como resultado de la alteración del estado de equilibrio termodinámico y químico (Cowan y Weintritt, 1976)

Remover las incrustaciones de un pozo es bastante costoso; además hay que agregar el costo de la producción demorada; por lo tanto, mantener los pozos productores en buen estado, constituye en definitiva, la forma más eficiente de producir hidrocarburos.

En la mayoría de los casos, para mantener la productividad de los pozos se prefiere utilizar el método de inhibición química como medio para prevenir la formación de incrustaciones. Las técnicas de inhibición pueden variar desde métodos básicos de dilución, a los más avanzados y efectivos inhibidores que actúan antes de que se inicie el proceso (Crabtree et al, 1999).

\section{Metodología}

Este proyecto fue desarrollado mediante el modelamiento de la predicción y tratamiento de las incrustaciones de carbonato de calcio; se elaboró un software llamado Scale Inhibition CaCO3, diseñado y programado exclusivamente para la aplicación en campos petroleros y es amplio en la predicción, control, tratamiento y manejo de las incrustaciones de carbonato de calcio en campos petroleros. A continuación se expone el estado del arte de la ciencia y la tecnología para el modelamiento de la predicción y tratamiento de las incrustaciones de carbonato de calcio.

\subsection{Predicción de la Tendencia a Formar Incrustaciones de Carbonato de Calcio}

Los valores del índice de saturación, SI, se calcularon sobre la base del algoritmo de Langelier LSI (Langelier ,1946) (Rafferty 1999).

El índice de Langelier (ó índice de saturación de carbonato de calcio), es importante para predecir las tendencias a la incrustación de carbonato de calcio del agua asociada al petróleo. Para calcular el índice de saturación de Langelier es necesario conocer los siguientes datos del agua de producción:

- Alcalinidad al metil-naranja

- Dureza cálcica

- Sólidos disueltos totales (TDS).

- $\mathrm{pH}$

- Temperatura del agua.

El índice de Langelier proporciona un valor cualitativo sobre la estabilidad del agua, aplicando la diferencia entre el pH (medido) y el pHs (equilibrio o saturación):

$\mathrm{LSI}=\mathrm{pH}-\mathrm{pHs}$

EL valor de pHs se puede obtener de la siguiente ecuación:

$\mathrm{pHs}=(9.3+\mathrm{A}+\mathrm{B})-(\mathrm{C}+\mathrm{D})$

Dónde:

$A=\log (T D S)-1$

$B=\left(-13.12 \log \left[\frac{{ }^{\circ} \mathrm{F}-32}{1.8}+273.15\right]\right)+34.55$

$\mathrm{C}=\log \left(\right.$ Dureza de $\left.\mathrm{Ca}^{++}\right)-0.4$ 
$\mathrm{D}=\log ($ Alcalinidad $\mathrm{M})$

Dónde: TDS :ppm

Dureza de $\mathrm{Ca}+2:$ ppm $\mathrm{CaCO}_{3}$

Alcalinidad $\mathrm{M}$ :ppm $\mathrm{CaCO}_{3}$

Si el índice de saturación de Langelier es igual a cero, el agua está en equilibrio químico, si es positivo es incrustante, y si es negativo es corrosiva.

\subsection{Selección y Requerimientos del Inhibidor}

La selección del inhibidor entre muchas opciones comerciales, y el cálculo de su mínima concentración efectiva necesaria son a menudo parámetros críticos en el tratamiento de incrustaciones. Los tipos de inhibidores comunes incluyen diversos aminopolifosfonatos, poliacrilatos, polisulfonatos, sus derivados y mezclas. El cálculo de la inhibición en este programa se basa en un modelo semiempírico de inhibición de la Nucleación. (He, Kan and Tomson. 1995) Los inhibidores disponibles en este programa son: NTMP [ácido nitrilotri (metileno fosfónico)], HDTMP [ácido hexametilendiamino tetra(metileno fosfónico)], DTPMP [ácido dietilentriamina penta(metileno fosfónico)], BHPMP [ácido bis-hexametilentriamina penta (metileno fosfónico)], PAA (ácido poliacrílico), PPCA (ácido fosfinopolicarboxílico) y SPA (ácido poliacrílico sulfonatado).

El conjunto de parámetros del modelo se limita a la calcita, la incrustación de yacimientos petrolíferos más común. La eficiencia de los inhibidores se ha modelado en función de las condiciones de campo, como el índice de

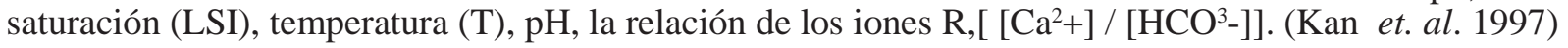

$C_{\text {inh }}(m g / L)=\frac{1}{b_{\text {inh }}(1 / m g)} \log \left\{f_{\text {sofery }} \frac{t_{\text {inh }}(\mathrm{sec})}{t_{o}(\mathrm{sec})}\right\}$

$\log \left[\boldsymbol{b}_{\text {inh }}(\mathrm{L} / \mathrm{mg})\right]=\beta_{\mathrm{o}}+\beta_{1} \bullet L \mathrm{SI}+\frac{\beta_{2}}{T(K)}+\beta_{2} \bullet \mathbf{p H}+\beta_{4} \bullet \log \boldsymbol{R}$

$\log _{o}\left[\mathrm{CaCo}_{2}, \mathrm{sec}\right]=4.22-\frac{13.8}{L S I}-\frac{1876.4}{-6259.6}$

Dónde:

Cinh Concentración mínima efectiva necesaria del inhibidor.

fsafety Factor de seguridad, generalmente se ajusta entre dos y tres.

binh Eficiencia de inhibición.

tinh Periodo de tiempo para que el sistema sea protegido de la formación de incrustaciones.

to Tiempo de inducción para que la solución sobresaturada pueda nuclear sin inhibidores.

$\beta$ o a $\beta 4$ Constantes enumeradas en la tabla 1 para la calcita.

Tabla 1. Coeficientes empíricos usados en el modelo de inhibición de la nucleación

\begin{tabular}{|c|c|c|c|c|c|}
\hline INHIBIDOR & $\boldsymbol{\beta O}$ & $\boldsymbol{\beta 1}$ & $\boldsymbol{\beta 2}$ & $\boldsymbol{\beta 3}$ & $\boldsymbol{\beta 4}$ \\
\hline BHPMP & -3.21 & -1.45 & 1918.7 & 0 & 0.27 \\
\hline NTMP & -0.53 & -1.61 & 1226 & 0 & 0.13 \\
\hline HEDP & 0.23 & -1.69 & 1082 & 0 & 0.14 \\
\hline HDTMP & -3.15 & -1.22 & 1813.1 & 0 & 0.27 \\
\hline DTPMP & -1.53 & -1.63 & 1396.4 & 0 & 0.29 \\
\hline PAA & -2.54 & -1.55 & 1734.6 & 0 & 0.27 \\
\hline SPA & -2.96 & -1.59 & 1768.8 & 0 & 0.2 \\
\hline PPCA & -3.04 & -1.41 & 1745.3 & 0 & 0.33 \\
\hline
\end{tabular}


En general, la eficiencia del inhibidor se expresa en términos de sus valores de binh a una condición dada, como una función de las variables de campo tales como el pH, T, LSI, y R en la ecuación (8). La selección de los inhibidores se basa en los valores calculados de binh para la incrustación de calcita a una condición dada. En general, cuanto mayor sea el valor de binh, más eficiente es el inhibidor. Por lo tanto, el inhibidor que exhiba el máximo binh será elegido. Además, el inhibidor puede ser seleccionado manualmente.

\subsection{Desplazamiento, Distribución y Colocación del Inhibidor}

Los tratamientos "squeeze" con inhibidores de incrustaciones en fondo del pozo son comunes en el planeamiento y control en muchas operadoras de campos petroleros. Sin embargo, las formaciones del yacimiento son largos cuerpos de rocas heterogéneas y el flujo de fluidos es fuertemente determinado por la permeabilidad de estas estructuras. Así, cuando se inyecta lentamente el inhibidor de incrustaciones en la formación, la colocación del fluido es un reto importante. Para diseñar exitosamente un tratamiento "squeeze", es necesario saber a dónde va a ir el fluido inyectado o, aún más, poder controlar la ubicación del fluido en la formación del yacimiento y en la cara del pozo. (Sorbie and Mackay. 20057)

Mediante este modelo se deriva la expresión analítica que describe la colocación en los sistemas de capas lineales y radiales para unidades de movilidad y fluidos viscosos. Se muestran cálculos sobre la colocación del inhibidor cerca al "wellbore” en sistemas radiales y lineales, mostrando cómo la teoría se acerca a un verdadero campo petrolero de sistemas heterogéneos.

Justificación del modelo. Los inhibidores químicos de incrustaciones se han aplicado durante muchos años en el fondo del pozo con tratamientos "squeeze”. El objetivo es contar con una concentración de retorno del inhibidor de incrustación en fase acuosa por encima de alguna concentración mínima de inhibidor (MIC), durante el mayor tiempo posible. El tiempo de vida del "squeeze" es función de la relación entre el inhibidor de incrustaciones y la roca, por ejemplo, la interacción por adsorción. En un depósito de capa homogénea, la adsorción puede ser el único mecanismo de retención que rige la devolución del inhibidor de incrustaciones desde el pozo. Sin embargo, rara vez las formaciones del yacimiento son homogéneas, están compuestas de rocas muy heterogéneas que pueden tener una o más capas de estructura compleja, determinada por diversas sedimentologías, factores estructurales y dia genéticos. Para efectos de este modelamiento se consideran únicamente los sistemas en los cuales las diversas capas tienen diferentes permeabilidades (k) y porosidades (Ø) en la formación cercana al pozo. En tales sistemas, la colocación del inhibidor de incrustaciones dentro de la formación es un aspecto adicional del tratamiento "squeeze" que debe ser considerado, ya que ésto puede afectar los retornos del inhibidor. En la mayoría de los casos, los inhibidores de incrustaciones se aplican como soluciones acuosas a una concentración, por lo general en un rango de 10000 a $150000 \mathrm{ppm}$. Éstas soluciones suelen tener una viscosidad $(\mu)$ próxima a la de una salmuera normal de inyección, es decir, $\approx 1 \mathrm{cP} \mathrm{a} 20^{\circ} \mathrm{C}\left(68^{\circ} \mathrm{F}\right)$ y $0,3 \mathrm{cP}$ a $100^{\circ} \mathrm{C}\left(212^{\circ} \mathrm{F}\right)$. Por lo tanto, además de un ligero efecto de la temperatura, la salmuera inyectada desplaza agua de formación en unidades de movilidad. También, para aceites ligeros, una unidad de movilidad es a menudo desplazada, aunque los efectos de viscosidad y permeabilidad pueden ser más importantes en los aceites más pesados.

En la inyección, la unidad de movilidad en una capa heterogénea para sistema lineal o radial se muestra esquemáticamente en la figura 1.

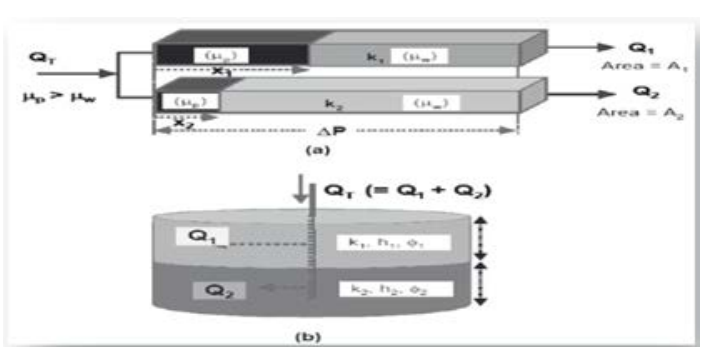

Figura 1. (a) Sistema de capas lineales heterogéneo (b) Sistema de capas radiales heterogéneo 
Sistemas lineales heterogéneos - sin flujo cruzado entre capas. El flujo de una unidad de movilidad de líquido es regido por la capa (kA)i. Para la colocación de líquidos viscosos, se usa la notación en la figura 1, en donde 1 y 2 son las capas de alta y baja permeabilidad respectivamente. Para una rata de inyección volumétrica constante de fluido, QT (QT = Q1 + Q2), para un fluido viscoso de viscosidad, $\mu \mathrm{p}(\mu \mathrm{p}>\mu \mathrm{w})$, el volumen acumulado se inyecta en el tiempo $t$ y viene dado por:

$$
\text { qinj }(\mathrm{t})=\mathrm{QT} \mathrm{t}
$$

La relación de flujo de la capa, (Q1/ Q2), puede ser demostrada y está dada por:

$$
\frac{Q_{1}}{Q_{2}}=\left[\frac{K_{1} \cdot A_{1}}{K_{2} \cdot A_{2}}\right]\left[\frac{\mu_{w} \cdot L+\left(\frac{\mu_{p}-\mu_{w}}{A_{2} \cdot \phi_{2}}\right)\left(q_{i n j}(t)-x_{1} A_{1} \phi_{1}\right)}{\mu_{w} \cdot L+\left(\mu_{p}-\mu_{w}\right) x_{1}}\right]
$$

Puesto que, $\mathrm{Q}_{\mathrm{T}}=\mathrm{Q}_{1}+\mathrm{Q}_{2}$, la tasa de avance en la capa 1 de alta permeabilidad está dada por:

$$
\frac{d x_{1}}{d t}=\frac{Q_{T} \cdot f_{1}\left(x_{1} ; t\right)}{A_{1} \phi_{1}\left(\frac{k_{2} \cdot A_{2}}{k_{1} \cdot A_{1}}+f_{1}\left(x_{1} ; t\right)\right)}
$$

Dónde:

$$
f_{1}\left(x_{1} ; t\right)=\frac{\mu_{w} \cdot L+\left(\frac{\mu_{p}-\mu_{w}}{A_{2}-\phi_{2}}\right)\left(\boldsymbol{q}_{i n j}(t)-x_{1} A_{1} \phi_{1}\right)}{\mu_{w} \cdot L+\left(\mu_{p}-\mu_{w}\right) x_{1}}
$$

L : Longitud máxima de penetración.

$\mathrm{X}$ : Distancia de avance en la capa 1.

A : Área transversal de flujo a los perforados en la capa 1.

A : Área transversal de flujo a los perforados en la capa 2.

El caudal o rata de flujo en cada una de las capas está dado por las ecuaciones 13 y 14.

$Q_{1}=\frac{\xi Q_{t}}{(1+\xi)}$

Dónde:

$$
\begin{gathered}
\xi=(\mathrm{Q} 1 / \mathrm{Q} 2) \\
\mathrm{Q}_{2}=\mathrm{Q}_{\mathrm{T}}-\mathrm{Q}_{1}
\end{gathered}
$$

La tasa de avance en la capa de baja permeabilidad puede ser fácilmente encontrada por balance de materia. El siguiente algoritmo se sigue para resolver la colocación del inhibidor de las ecuaciones anteriores: 
1. Hacer que (x1) viejo sea igual a cero.

2. Para $\mathrm{t}=0, f l=1$; calcular $\left(\mathrm{dx}_{1} / \mathrm{dt}\right)$ de ecuación 12 .

3. Actualizar $\rightarrow\left(\mathrm{x}_{1}\right)$ nuevo $=\left(\mathrm{x}_{1}\right)$ viejo $+\Delta \mathrm{t}^{*}\left(\mathrm{dx}_{1} / \mathrm{dt}\right)$

4. Calcular $\left(\mathrm{Q}_{1} / \mathrm{Q}_{2}\right)$ de ecuación 11 .

5. Calcular $\left(\mathrm{Q}_{1}\right)$ de ecuación 13.

6. Calcular $\left(\mathrm{Q}_{2}\right)$ de ecuación 14

7. Actualizar tiempo para $\mathrm{t}=\mathrm{t}+\Delta \mathrm{t}$

8. Hacer que $\left(\mathrm{x}_{1}\right)_{\text {viejo }}$ sea igual a $\left(\mathrm{x}_{1}\right)_{\text {nuevo }}$

9. Ir al paso 2 .

La iteración termina cuando $\left(\mathrm{x}_{1}\right)$ nuevo sea igual a la longitud máxima de penetración L.

Sistemas radiales heterogéneos - sin flujo cruzado entre capas. Para la colocación de una unidad de movilidad $(\mu \mathrm{p}=\mu \mathrm{w})$ en un sistema radial, la relación de caudal está dado por la siguiente ecuación:

$$
\begin{aligned}
& \frac{Q_{1}}{Q_{2}}=\left[\frac{k_{1} \cdot h_{1}}{k_{2} \cdot h_{2}}\right] \\
& \frac{r_{1}}{r_{2}}=\sqrt{\frac{k_{1}}{k_{2}}} \\
& \frac{Q_{1}}{Q_{2}}=\frac{k_{1} \cdot h_{1}}{k_{2} \cdot h_{2}} \cdot \frac{\ln \left(\frac{R_{\max } \cdot r_{1}^{(\beta-1)}}{r_{w}{ }^{\beta}}\right)}{\ln \left(\frac{R_{\max } \cdot r_{2}{ }^{(\beta-1)}}{r_{w}{ }^{\beta}}\right)}
\end{aligned}
$$

Donde:

$\beta=\frac{\mu_{p}}{\mu_{w}}$

Rmax: Radio máximo de penetración.

$\mathrm{r}_{1}$ : Radio de avance en la capa 1.

r2: Radio de avance en la capa 2.

$\mathrm{r}_{\mathrm{w}}$ : Radio del pozo.

$\mathrm{K}_{1}$ : Permeabilidad de la capa 1.

$\mathrm{K}_{2}$ : Permeabilidad de la capa 2.

$\mathrm{h}_{1}$ : Espesor de los perforados en la capa 1.

$\mathrm{h}_{2}$ : Espesor de los perforados en la capa 2.

De igual manera que en sistemas lineales heterogéneos, el caudal o rata de flujo del inhibidor puede ser evaluado con las siguientes ecuaciones:

$\boldsymbol{Q}_{1}=\frac{\xi \boldsymbol{Q}_{T}}{(\mathbf{1}+\xi)}$

Donde:

$\xi=\left(Q_{1} / Q_{2}\right)$ 


$$
\mathrm{Q}_{2}=\mathrm{Q}_{\mathrm{T}}-\mathrm{Q}_{1}
$$

El radio de avance frontal puede ser calculado en función del tiempo por medio de las siguientes ecuaciones:

$$
\begin{aligned}
& \left(r_{1}\right)_{\text {nuevo }}=\left(\frac{Q_{1 . \Delta t}}{\pi h_{1 . \phi 1}}+\left(r_{1}\right)_{\text {viejo }}^{2}\right)^{1 / 2} \\
& \left(r_{1}\right)_{\text {nuevo }}=\left(\frac{Q_{1 . \Delta t}}{\pi h_{1 . \phi 1}}+\left(r_{1}\right)_{\text {viejo }}^{2}\right)^{1 / 2}
\end{aligned}
$$

Donde:

$\Delta_{\mathrm{t}}=$ Intervalo de tiempo

$\Phi_{1}^{\mathrm{t}}=$ Porosidad de la capa 1

$\Phi_{2}=$ Porosidad de la capa 2

El algoritmo para resolver el avance frontal en cada capa, se expone a continuación:

\section{Resultados}

1. Hacer que $\left(\mathrm{r}_{1}\right)$ viejo y $\left(\mathrm{r}_{2}\right)$ viejo sean iguales al radio del pozo $\mathrm{r}_{\mathrm{w}}$.

2. Calcular $\left(\mathrm{Q}_{1} / \mathrm{Q}_{2}\right)$; para el tiempo inicial $(\mathrm{t}=0)$ se calcula con la ecuación 15 y para $\mathrm{t}>0$ con la ecuación 17.

3. Calcular $\mathrm{Q}_{1}$ con la ecuación 18.

4. Calcular $\mathrm{Q}_{2}$ con la ecuación 19.

5. Calcular $\left(\mathrm{r}_{1}\right)$ nuevo y $\left(\mathrm{r}_{2}\right)$ nuevo con las ecuaciones 20 y 21 respectivamente.

6 . Hacer que $\left(\mathrm{r}_{1}\right)$ viejo $=\left(\mathrm{r}_{1}\right)$ nuevo y $\left(\mathrm{r}_{2}\right)_{\text {viejo }}=\left(\mathrm{r}_{2}\right)_{\text {nuevo }}$

7. Actualizar tiempo para $\mathrm{t}=\mathrm{t}+\Delta \mathrm{t}$

8. Ir al paso 2.

La iteración termina cuando $\left(\mathrm{r}_{1}\right)$ nuevo sea igual a radio máximo de penetración $\mathrm{R}_{\mathrm{m}}$

- Eficiencia del inhibidor. La eficiencia de los inhibidores se evaluó a diferentes temperaturas para el pozo SF-030, en un rango de $50^{\circ} \mathrm{F}\left(10^{\circ} \mathrm{C}\right)$ a $350^{\circ} \mathrm{F}\left(177^{\circ} \mathrm{C}\right)$, con el fin de simular situaciones críticas de producción en superficie a bajas temperaturas y sistemas de inyección de vapor sobrecalentado.

En ambientes de producción de hasta $150^{\circ} \mathrm{F}$, los inhibidores exhiben una eficiencia relativamente aceptable que puede variar desde 1,69-611,3 L/mg para el PPCA hasta 20,90 - 5981,42 L/mg para el HEDP; en la figura 2 se ilustra dicho comportamiento. 


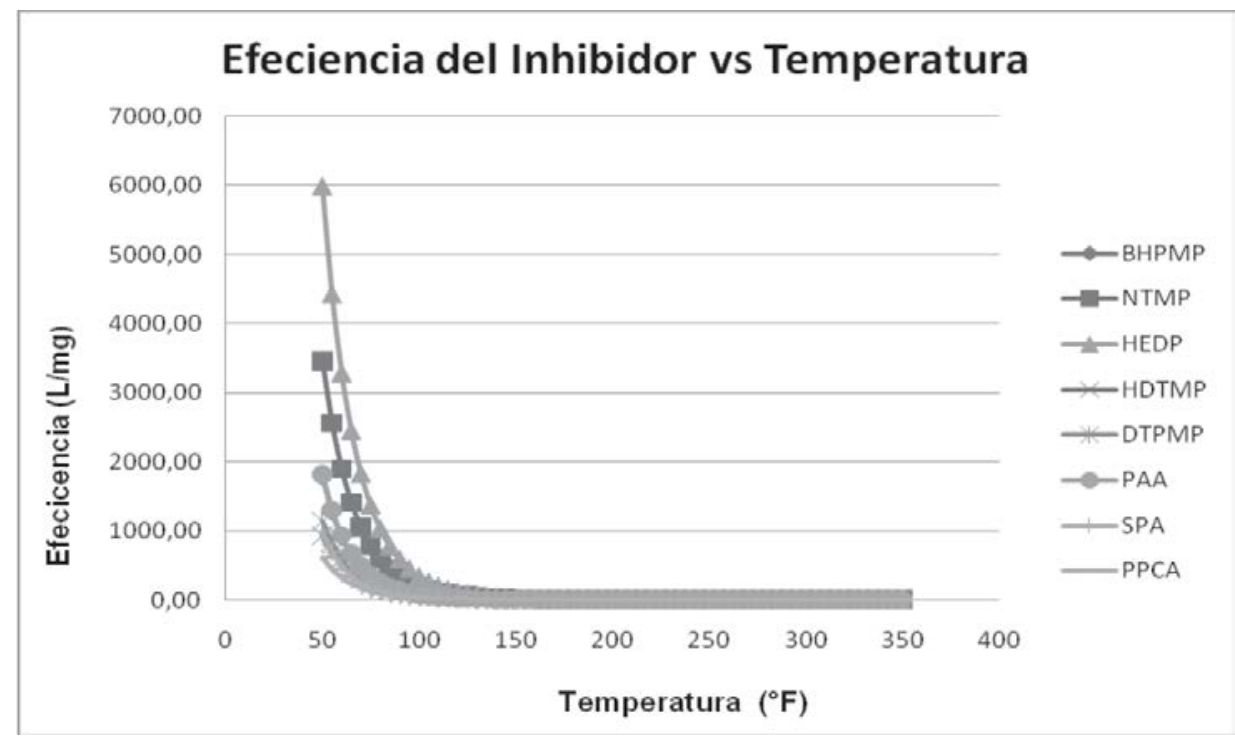

Figura 2. Eficiencia de inhibición para diferentes inhibidores de incrustaciones para un rango de temperatura de 50 a $350^{\circ} \mathrm{F}$.

En yacimientos con temperaturas por encima de los $150^{\circ} \mathrm{F}$ la inhibición del carbonato de calcio se torna crítica mediante los inhibidores disponibles en el programa. Aunque la eficiencia de inhibición es fuertemente influenciada por el $\mathrm{pH}$ y la composición del agua de producción, la temperatura del yacimiento es un parámetro modificador y constante.

- Penetración y colocación del inhibidor. En el presente estudio se muestra la penetración y colocación del inhibidor HEDP en un sistema radial homogéneo-isotrópico (pozo SF-042) y en un sistema de capas radial heterogéneo-anisotrópico (pozo SF-030).

- Sistema de capas radial heterogéneo-anisotrópico (pozo SF-030). Los parámetros petrofísicos del Pozo SF030 se muestran en la tabla 7 y fueron utilizados en el cálculo de la penetración y colocación del inhibidor. El radio de tratamiento de inhibición y la rata de inyección fueron de 5 pies y 3 BPM respectivamente.

Como se muestra en la tabla 2, el pozo SF-030 presenta dos zonas productoras correspondientes a la formación Caballos. La capa de alta permeabilidad corresponde al estrato KCU y la capa de baja permeabilidad corresponde al estrato KCL. La figura 3 muestra que en KCU el radio de penetración avanza a mayor velocidad que en KCL, por consiguiente la píldora del inhibidor inyectado se fijará en primera instancia en el radio máximo de penetración en KCU, luego lo hará en KCL.

Tabla 2. Parámetros petrofísicos del pozo SF-030

\begin{tabular}{|c|c|c|}
\hline \multicolumn{1}{|c|}{ Pozo } & \multicolumn{2}{|c|}{ SF-030 } \\
\hline Capa & KCU & KCL \\
\hline Permeabilidad (mD) & 1800 & 862 \\
\hline Porosidad (\%) & 15 & 10 \\
\hline Espesor(pies) & 91 & 35 \\
\hline $\begin{array}{c}\text { Ancho o Radio del pozo } \\
\text { (pies) }\end{array}$ & \multicolumn{2}{|c|}{0,5} \\
\hline
\end{tabular}

Si la diferencia entre permeabilidades es muy alta, el radio de penetración del inhibidor en la zona de baja permeabilidad será menor al proyectado en el programa de inyección. 
En el caso de KCU, los 5 pies de penetración proyectados se alcanzaron a los 1780 minutos (29.67 horas), tiempo para el cual el radio de penetración de KCL fue 4.18 pies. Para que KCL alcance el radio de penetración proyectado se deberá fijar un radio de inhibición de 6 pies.

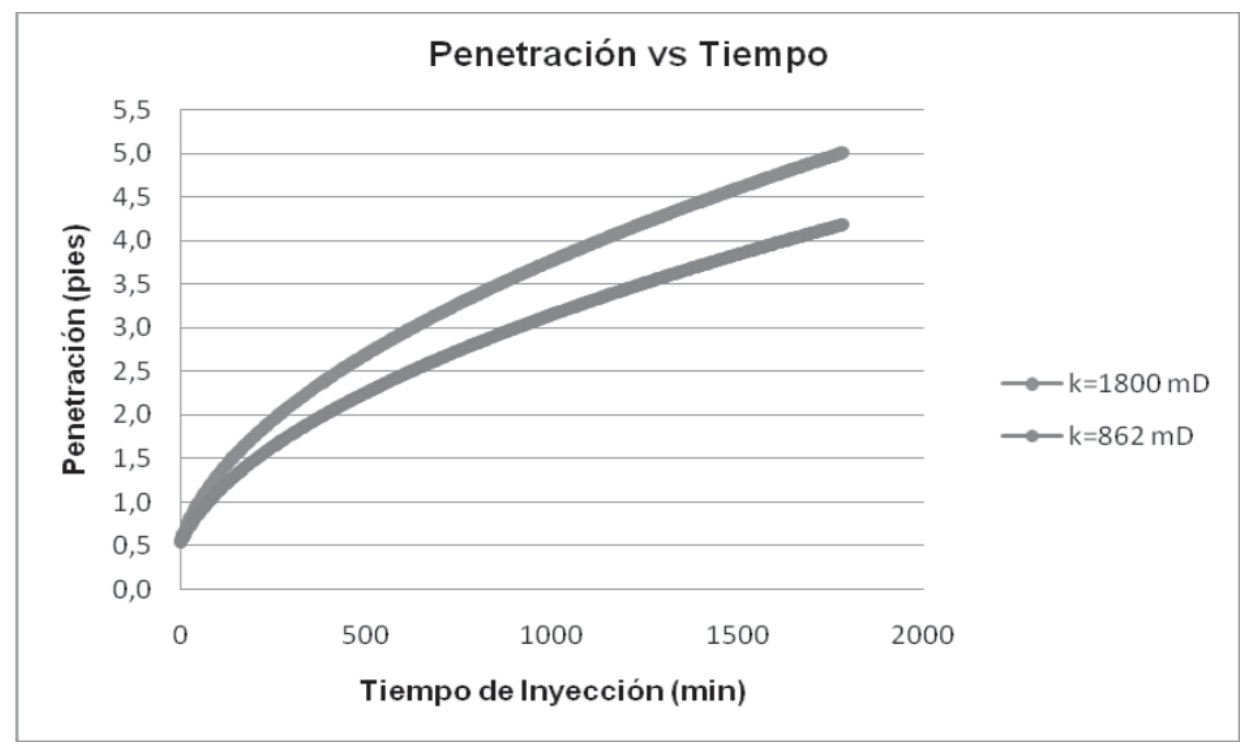

Figura 3. Aumento del radio de penetración en función del tiempo.

Sistema de capas radial homogéneo-isotrópico (Pozo SF-042). En el caso del Pozo SF-042, el tratamiento de inhibición se hizo solamente en la capa KCU lo cual convierte el sistema en homogéneo-isotrópico. Los parámetros petrofísicos del pozo se muestran en la tabla 3. Para poder simular la colocación y penetración del inhibidor en KCU, se creará a un sistema equivalente de dos capas como se muestra en la tabla 4, conservando las propiedades petrofísicas del sistema original. Al igual que en SF-030, el radio de tratamiento de inhibición y la rata de inyección fueron de 5 pies y 3 BPM respectivamente.

Tabla 3. Parámetros petrofísicos capa KCU

\begin{tabular}{|l|c|}
\hline \multicolumn{1}{|c|}{ Pozo } & SF- 042 \\
\hline Capa & KCU \\
\hline Permeabilidad (mD) & 1800 \\
\hline Porosidad (\%) & 16 \\
\hline Espesor(pies) & 64 \\
\hline Radio del pozo (pies) & 0,5 \\
\hline
\end{tabular}

Tabla 4. Sistema equivalente de la del pozo de la SF-042

\begin{tabular}{|l|c|c|}
\hline \multicolumn{1}{|c|}{ Pozo } & \multicolumn{2}{|c|}{ SF-042 } \\
\hline Capa & KCU & KCU \\
\hline Permeabilidad (mD) & 1800 & 1800 \\
\hline Porosidad (\%) & 16 & 16 \\
\hline Espesor(pies) & 32 & 32 \\
\hline Radio del pozo (pies) & \multicolumn{2}{|c|}{0,5} \\
\hline
\end{tabular}




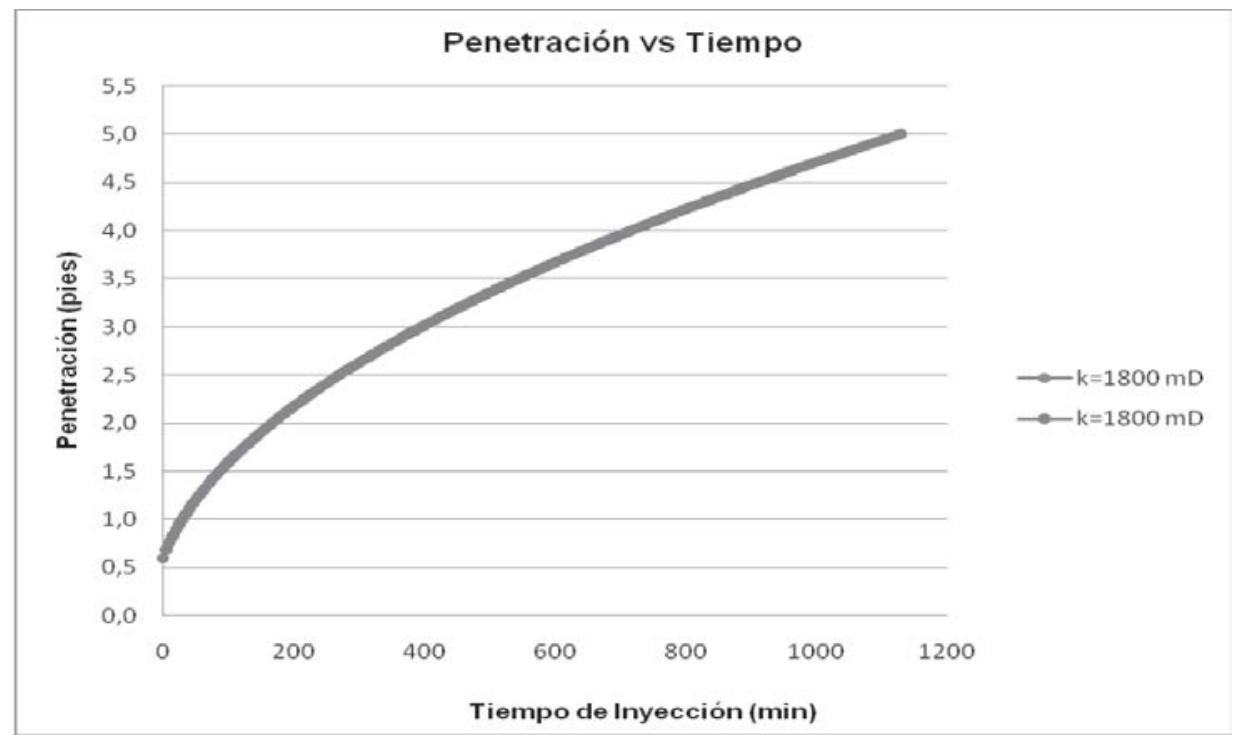

Figura 4. Aumento del radio de penetración en función del tiempo para un sistema homogéneo-isotrópico.

El radio de penetración para un sistema equivalente de permeabilidades se muestra en la figura 64 Las curvas de penetración se superponen para este tipo de sistemas mostrando un solo radio de avance equivalente al sistema original.

\section{Conclusiones}

Se modeló termodinámicamente la inhibición de incrustaciones por carbonato de calcio en sistemas de hidrocarburos. El cálculo de la inhibición en este programa se basa en un modelo semiempírico de inhibición de la nucleación.

Se modeló mediante el análisis del flujo de fluidos en medios porosos, la colocación y el radio de penetración del inhibidor en la formación con el fin de monitorear el tiempo de inyección y optimizar los tratamientos "squeeze”.

Los inhibidores evaluados muestran eficiencias aceptables hasta $150^{\circ} \mathrm{F}$. En sistemas de producción que operan por encima de este valor, la inhibición de incrustaciones de carbonato de calcio se torna crítica y costosa.

La concentración mínima del inhibidor necesaria para que se inhiba la incrustación de carbonato de calcio exhibe una buena confiabilidad en el dato arrojado por el programa Scale Inhibition CaCO3. Además, se puede observar la similitud con los resultados obtenidos por el programa SqueezeSoftPitzer.

Scale Inhibition CaCO3 puede ser aplicado en campos petroleros que manejen aguas de producción o inyección con concentraciones de hasta $300000 \mathrm{mg} / \mathrm{L}$ en sólidos totales disueltos (TDS), en un rango de temperaturas entre 50 y $350^{\circ} \mathrm{F}$.

\section{Bibliografía}

1.Cowan, J.C. y Weintritt, J.D.1976. “Water - Formed Scale Deposits”. Chapter 1. Gulf Publishing.Texas.

2. Crabtree, Mike, et al. 1999, "La lucha contra las incrustaciones- Remoción y prevención”. Oilfield Review, Schlumberger, volumen 11, número 3. .

3. Langelier W.F. 1946. “Chemical Equilibrium in Water”. JAWWA, Vol. 38.

4.Rafferty, K.1999. “Scaling in Geothermal Heat Pump Systems”. Oregon Institute of Technology, Julio de 
5. He, S.L., Kan, A.T. and Thomson, M.B.1995. "Mathematical inhibitor model for barium sulfate scale control”. Langmuir 12 , Pag 1901-1905.

6. He, S.L., Kan, A.T., Thomson, M. B. and Oddo, J. E.1997. “A New Interactive Software for Scale Prediction, Control, and Management”. SPE 38801. Presentado en la conferencia y exhibición técnica anual SPE. San Antonio, Texas. Octubre de 1997.

7. Sorbie, K.S. and Mackay, E.J.2005. “Scale Inhibitor Placement: Back to Basics-Theory and Examples”. SPE 95090. 